\title{
Questes
}

\section{L'art de prêcher la faute : rhétorique et esthétique dans les sermons de Jean Gerson}

\section{Viviane Griveau-Genest}

\section{(2) OpenEdition}

\section{Journals}

\section{Édition électronique}

URL : http://journals.openedition.org/questes/4248

DOI : 10.4000/questes.4248

ISSN : 2109-9472

\section{Éditeur}

Les Amis de Questes

\section{Édition imprimée}

Date de publication : 30 octobre 2015

Pagination : 79-93

ISSN : 2102-7188

\section{Référence électronique}

Viviane Griveau-Genest, «L'art de prêcher la faute : rhétorique et esthétique dans les sermons de Jean Gerson », Questes [En ligne], 30 | 2015, mis en ligne le 01 janvier 2015, consulté le 30 avril 2019. URL : http://journals.openedition.org/questes/4248; DOI : 10.4000/questes.4248 


\title{
L'art de prêcher la faute : rhétorique et esthétique dans les sermons de Jean Gerson
}

\author{
Viviane GRIVEAU-GENEST \\ Université Paris Ouest Nanterre La Défense - UNIGE
}

Qu'est-ce que la prédication médiévale? Alain de Lille nous renseigne dans sa Summa Praedicatoria: «la prédication est un enseignement public traitant des mœurs et de la foi, destiné à informer les hommes. Elle emprunte la voie de la raison et elle tire sa source des autorités ${ }^{1}$. » Cette définition canonique au Moyen Âge permet de saisir des traits majeurs des sermons d'alors : il s'agit avant tout d'un discours pédagogique, centré sur des considérations religieuses ${ }^{2}$. Ce faisant, Alain de Lille reste discret sur le statut générique du texte en question. Cette théorisation pragmatique de la parole homilétique est la plus communément répandue à partir $\mathrm{du} \mathrm{XIII}^{\mathrm{e}}$ siècle dans les milieux où émerge la prédication dite «scolastique ${ }^{3}$ » ainsi que dans les artes praedicandi ${ }^{4}$. Dans cette perspective, le prédicateur est un ouvrier de la

1 Alain de Lille, Summa Praedicatoria, «Praedicatio est, manifesta et publica instructio morum et fidei, informationi hominum deserviens, ex rationum semita, et auctoritatum fonte proveniens », Patrologie Migne, vol. 210, coll. 109.

2 Le terme est vaste à dessein: un sermon peut être en effet centré sur des considérations dogmatiques ou théologiques, sur des considérations morales ou des considérations spirituelles. C'est sans compter l'importance des thématiques autres qui participaient alors de la vie chrétienne : politique, réflexions sociales etc.

${ }^{3}$ Voir Nicole Bériou, L'Avènement des maîtres de la Parole. La prédication à Paris au XIII siècle, Paris, Institut des Études Augustiniennes, 1998 et Constructing the medieval sermon, dir. Roger Andersson,Turnhout, Brépols, 2007.

${ }^{4}$ Sur la question des Artes praedicandi, voir Marianne Briscoe, Artes praedicandi, Turnhout, Brépols, coll. « Typologie des sources du Moyen Âge occidental », 1992 et Thomas-Marie Charland, Artes praedicandi. Contribution à l'histoire de la rhétorique au Moyen Âge, Ottawa/Paris, Institut d'études médiévales/Vrin, 1936. 
Parole, au sens théologique, et de la parole, au sens rhétorique, l'humble instrument, un calame, aux mains d'un scribe véloce, Dieu.

Nous adopterons ici une méthodologie sensiblement différente pour nous interroger précisément sur le statut de ce discours qu'est la prédication en posant, à la suite d'études récentes ${ }^{5}$, que la prédication n'est pas seulement un discours didactique et dogmatique mais aussi une forme esthétique caractéristique de la production culturelle du Moyen Âge, mêlant intellectualité, spiritualité et littérarité. Dans cette perspective, l'introduction de la récente synthèse The Sermon ${ }^{6}$ convoque la double référence de Jauss et Combe pour parler de « genre littéraire ». Prêcher la faute est donc tout un art et la forme, autant que le contenu, pouvait faire l'objet d'une appréciation esthétique : par-delà les artes, on retrouve ici la conception fondamentale pour la pensée médiévale du De Doctrina christiana ${ }^{7}$ qui scelle l'alliance de la foi et de la rhétorique.

La figure du chancelier de l'université de Paris qu'est Jean Gerson (1363-1429) offre à cet égard un cas d'école : d'une part, parce que la carrière de Gerson fut tributaire de ses succès comme prédicateur de cour, d'autre part en raison de ses préoccupations littéraires ${ }^{8}$ et enfin

5 Pour un aperçu d'un traitement interdisciplinaire des sermons médiévaux voir Modern Questions about Medieval Sermons. Essays on Marriage, Death, History, and Sexuality, dir. Nicole Bériou, et David d'Avray, Church History, vol. 66, $\mathrm{n}^{\mathrm{o}} 1$, p. 101-103 et Michel Zink, «L'Art littéraire de saint Bernard. Une esthétique de la conversion ", L'Actualité de Saint Bernard. Actes du colloque des 20 et 21 novembre 2009, dir. Antoine Guggenheim, André-Marie Ponnou-Delaffon, Paris, Lethielleux, 2010, p. 197-211.

${ }^{6}$ Voir Mayne Beverly Kienzle, The Sermon, Turnhout, Brépols, coll. « Typologie des sources du Moyen Âge Occidental », 2000.

${ }^{7}$ Augustin d'Hippone, De Doctrina christiana, éd. Goulven Madec, trad. Madeleine Moreau, Paris, Institut d'Études Augustiniennes, coll. «Bibliothèque augustinienne », 1997.

${ }^{8}$ Les écrits du chancelier ont fait récemment l'objet d'une édition intégrale en onze volumes: Jean Gerson, CEuvres complètes, éd. Mgr Palémon Glorieux, Paris, Desclée, 1960-1973, 10 t. en 11 vol. Si cette entreprise facilite notablement l'accès aux textes, précisons qu'elle n'en fournit pas toujours une édition scientifique rigoureuse. Pour la prédication, on pourra se reporter au tome $\mathrm{V}$ pour les textes latins, «L'Euvre Oratoire », 1963, que nous abrégerons dans la suite de notre 
du fait que ses sermons ont fait l'objet d'une diffusion à caractère auctorial $^{9}$. Souci esthétique du côté de l'auteur ${ }^{10}$, mais aussi réception esthétique du côté des auditeurs : c'est ce que montre le portrait fait par Jean de Montreuil du chancelier à Guillaume Fillastre :

«[..] miror et admodum miror te illius eruditionis singularissimae viri cancellarii Parisiensis non esse imitatorem. De vita non loquor aut moribus, seu etiam de cultu religionis christiniae vel de theologicae, in quibus electissimi elevatissimique ambo estis. De traditiva et suasiva loquor, quae maxime rethorices et eloquentiae regulis constat consequiturque, et sine qua sermocinatio, quae

article en $O . O$, et, pour les textes français, au tome vII. 2 «Sermons et discours », 1968, que nous abrégerons dans la suite de notre article en $O . F$. Les traductions des textes latins sont de notre main. Plus largement, concernant le champ des études gersoniennes, on consultera, sur la sensibilité littéraire de Jean Gerson, les textes où il se positionne en acteur du champ littéraire : pour ses écrits sur le Roman de la Rose, voir Christine de Pisan, Jean Gerson, Jean de Montreuil, Gontier et Pierre Col, Le Débat sur le "Roman de la Rose », éd. Éric Hicks, Paris, Champion, coll. «Bibliothèque du XV ${ }^{\mathrm{e}}$ siècle », 1977 ; pour un aperçu de ses écrits oratoires et théologiques, voir l'étude et l'édition du «Contre Juan de Monzon », Gilbert Ouy, «La plus ancienne œuvre retrouvée de Jean Gerson. Le brouillon inachevé d'un traité contre Juan de Monzon (1389-1390) », Romania, 83, 1962, p. 469. Sur ce dernier personnage, un dominicain aragonais, notons que la bibliographie accepte également l'orthographe «Juan de Monzon » que «Jean de Monzon ». Enfin, pour ce qui touche les écrits poétiques du chancelier, on se référera aux Euvres complètes, éd. Palémon Glorieux, t. IV, Paris, Desclée, 1962 et à l'étude de la Josephina par Matteo Roccatti, «La Josephina di Jean Gerson (1418) : un poema virgiliano di contenuto biblico », Studi francesi, 121, 1997, p. 3-19.

${ }^{9}$ Sur ces questions d'auctorialité, se référer au récent ouvrage de Daniel Hobbins Authorship and Publicity before Print, Philadelphia, University of Pennsylvania Press, 2009.

${ }^{10}$ Le terme pourrait ici passer pour impropre tant il est vrai que la notion médiévale d'auteur impose la nuance. Sur cette question, on se reportera entre autres, à Michel Zink, La Subjectivité littéraire. Autour du siècle de saint Louis, Paris, PUF, 1985, et à Daniel Hobbins, Authorship and Publicity before Print, op. cit. Sur la question du contexte intellectuel de la fin du Moyen Âge, voir Ezio Ornato, Jean Muret et ses amis Nicolas de Clamanges et Jean de Montreuil. Contribution à l'étude des rapports entre les humanistes de Paris et ceux d'Avignon (1394-1420), Genève, Droz, 1969, ainsi qu'à Yelena Mazour-Matusevitch, «Gerson et Pétrarque : humanisme et l'idée nationale », Renaissance et Réforme, vol. XXV, $\mathrm{n}^{\mathrm{o}} 1,2001$, p. 45-80. 
finis esse mihi videt facultatis, redditur pene inutilis et vacua. ${ }^{11}$

Qu'il nous suffise ici de souligner que Jean de Montreuil, chef de file du préhumanisme français, présente le chancelier comme un modèle esthétique, digne d'imitation du fait de sa maîtrise rhétorique, et non comme modèle d'ecclésiastique. Ainsi, les sermons de Jean Gerson brillaient par leur art rhétorique aux yeux des médiévaux : le «fairecroire $^{12} \gg$ supposait bien, en accord avec Alain de Lille, l'exposition de vérités doctrinales et morales mais il ne s'y cantonnait pas chez Gerson ; ici, praedicatio rime aussi avec delectatio. Toutefois, l'écriture n'est en rien gratuite ${ }^{13}$ : la rhétorique sert aussi à tempérer la morale amère par le miel du langage.

J'explorerai donc la dimension esthétique du sermon gersonien à travers trois axes : d'abord en montrant que la prédication de la faute convoque une réception de type esthétique par la mobilisation d'émotions ${ }^{14}$ chez l'auditeur ou le lecteur ${ }^{15}$. Nous verrons ensuite que

11 « [...] je suis étonné, du moins je m'étonne de ce que tu ne te fasses pas l'imitateur d'un homme d'une rare et singulière érudition, le chancelier de l'université de Paris. Je ne parle pas de sa vie ou de ses mœurs non plus que de son culte de la religion chrétienne ou de la théologie, choses où vous vous distinguez tous deux par votre élévation. Mais je parle de l'art de conter et de persuader qui reposent et suivent surtout les règles de la rhétorique et de l'éloquence et sans lesquelles la prédication, qui m'apparaît comme le sommet de l'art, est rendue pour ainsi dire inutile et vaine ». Cf. Jean Gerson, Euvres complètes, op. cit., t. I, 1960, p. 471. Nous nous sommes inspiré pour notre traduction de celle proposée par Jacques Le Goff dans Jacques Le Goff, Les Intellectuels au Moyen Âge, Paris, Le Seuil, 1957, p. 175.

${ }^{12}$ Faire croire. Modalités de la diffusion et de la réception des messages religieux du $X I I^{e}$ au XV $V^{e}$ siècle. Actes de la table ronde de Rome (22-23 juin 1979), Rome, École Française de Rome, 1981.

${ }^{13}$ Voir Michel Zink, Poésie et Conversion, Paris, PUF, 2003.

${ }^{14}$ Sur les émotions en contexte homilétique, on se reportera à l'article de Dyonisios Stathakopoulos, «Prêcher les émotions incarnées. Évêques, mendiants et leurs publics dans l'Antiquité tardive », Médiévales, $\mathrm{n}^{\circ}$ 61, automne 2011, mis en ligne le 17 janvier 2012, consulté le 18 février 2014. URL: http://medievales.revues.org/6250. Plus largement, on se référera à Piroska Nagy, Le Don des larmes au Moyen Âge. Un instrument en quête d'institution, Paris, 
l'usage des cadres littéraires permet de remodeler le contenu moral en accord avec la sensibilité culturelle de l'époque. Enfin, faire la morale avec art, ou faire de la morale un art suppose de jouer des ressources énonciatives du sermon pour moduler un discours qui dérange.

\section{Une construction esthétique : « travailler » l'auditeur-lecteur au cour}

Si le sermon est un genre littéraire, reconnaissons que le sousgenre du sermon universitaire ne met pas en avant les couleurs rhétoriques. À l'inverse d'autres prédications ${ }^{16}$, le sermon universitaire déploie une esthétique austère et pudique. Et pourtant Gerson ne réduit jamais son discours à la sphère intellectuelle : il s'agit «d'émouvoir à la vertu $^{17} »$, le sermon visant l'adhésion du destinataire en suscitant sa conversion. L'écriture informe ainsi le dogme de manière imageante ${ }^{18}$, en orchestrant les émotions, pour tracer un chemin d'affects vers la foi.

Albin Michel, 2000 et à Damien Boquet, L'Ordre de l'affect au Moyen Âge. Autour de l'anthropologie affective d'Aelred de Rievaux, Caen, Publications du CRAHM, 2005.

${ }^{15}$ Les sermons ont vocation à être prêchés mais aussi à être diffusés par écrit : le destinataire est donc soit auditeur soit lecteur. Nous utiliserons dans le reste de l'article « auditeur » sans préjuger de la variété des réceptions.

${ }^{16}$ Pour avoir un aperçu d'autres contextes homilétiques, on peut se référer à Don Jean Leclercq, L'Amour des lettres et le désir de Dieu, Paris, Le Cerf, 1957, et Wim Verbaal, Speculum Sermonis. Interdisciplinary Reflections on the medieval Sermon, Turnhout, Brépols, 2004, vol. I, p. 113-139.

${ }^{17}$ Les mots sont de Gerson au seuil du sermon, «Ad Deum vadit », $\mathrm{n}^{\mathrm{0}} 341$ de l'édition Palémon Glorieux, op. cit : «Et m'efforceray principalement a esmouvoir nos cueurs a devocion et a deuil de ceste tres angoisseuse passion. », p. 453.

${ }^{18}$ Il s'agit, par notre néologisme, de souligner la dimension proprement « vive » des images selon la belle formule de Paul Ricœur. Sur le rôle de l'image chez Gerson, voir Virginie Minet-Mahy, Esthétique et pouvoir de l'œuvre allégorique à l'époque de Charles VI. Imaginaires et discours, Paris, Champion, 2005 et Jean Gerson, La Doctrine du chant du cour. Édition critique, traduction et commentaire $d u$ Tractatus de canticis et du Canticordum au pèlerin, éd. Isabelle Fabre, Genève, Droz, 2005. 
Maniant la peur ${ }^{19}$, ou le désir du bien, le prédicateur guide le fidèle vers son salut. Ce sont donc d'abord les figures, métaphores et allégories ${ }^{20}$, qui font un texte séduisant. La métaphore est le maître outil pour donner corps et saveur au dogme, comme ici, la métaphore musicale ${ }^{21}$ qui fait résonner l'harmonie régnant dans l'âme vertueuse :

[...] luctus et consolatio eamdem animam tanta celeritate alternatim invicem afficiunt quantam discernere non valemus; et consequenter poneretur motus cordis quasi tremulus et mixtus ex constrictione et dilatatione, forte similis motui campanae dum tremit, et chordae citharae dum resonat; et ita mirabilis harmonia fit in corde nostro ex hac mutua percurssione luctus et consolationis, qualem harmoniam explicare sicut difficile est, ita experiri facile ${ }^{22}$.

Ici, c'est toute la musicalité de la langue latine médiévale que Gerson convoque pour faire vibrer l'image. La métaphore peut aussi servir de repoussoir par le biais d'associations prosaïques :

Homo [...] comparatus est jumentis insipientibus et similis factus est illis. Sequimur eum nos bestiales effecti, vitam pecudum eligentes qui declivem jugiter ad terrena animam demittimus,

${ }^{19}$ Voir Jean Delumeau, Le Péché et la peur. La culpabilisation en Occident (XIII ${ }^{e}$ XVIII $e^{e}$ siècles), Paris, Fayard, 1983. Soulignons toutefois, à la suite de Louis Mourin dans Jean Gerson, prédicateur français, Brugge, De Tempel, 1952, que la prédication de la peur est limitée chez Gerson.

20 Voir Armand Strubel, Allégorie et littérature au Moyen Âge. "Grant senefiance $a »$, Paris, Honoré Champion, 2002.

${ }^{21}$ La métaphore musicale est propre à la sensibilité gersonienne : ponctuelle dans les sermons à l'inverse des écrits lyonnais. Voir Jean Gerson, La Doctrine du chant $d u$ cour, op. cit.

22 «Le deuil et la consolation affectent successivement cette même âme avec une telle rapidité que nous sommes impuissants à le voir. De sorte que, pour ainsi dire, le mouvement du cœur est analogue à une vibration mêlée de contrition et de dilatation, sans doute à rapprocher du mouvement de la cloche qui vibre et des cordes de la cithare qui résonne. Ainsi se produit une harmonie admirable dans notre cœur par cette mutuelle percussion de deuil et de consolation, laquelle harmonie est aussi difficile à expliquer qu'elle est facile à ressentir. » $O . O$., $n^{\mathrm{o}} 213$, p. 99. 
qui porcino more versis ad infima vultibus adharemus ${ }^{23}$.

C'est l'art rhétorique du prédicateur qui transforme ici ce topos de la pensée religieuse en véritable vision. Le jeu subtilement rythmique des syntagmes : comparatus est /5/ jumentis /3/ insipientibus /5/ et similis /3/ factus est /3/ illis /2/ et l'entrelacement des sonorités en une ligne mélodique continue permettent la mise en valeur de l'accusation. Ainsi l'association de l'homme à la bête vile et sans âme peut-elle devenir une ressource au service de la persuasion morale.

Si le prédicateur fait parfois appel au sens esthétique de l'auditeur pour prêcher la faute, il peut également mobiliser le charme des realia pour capter l'attention. Ici, c'est donc l'image du ménage qui doit exciter à la pénitence :

[...] pour recevoir dignement ce noble et si glorieux hoste, comme le Saint Esperit, s'efforça en droit soy d'appareiller l'ostel de son ame et de son espirituelle habittation, tellement que n'y fust trouvee la poudre d'avarice, la fiente de luxure, les araines d'envie et d'ire et ne la fumee d'orgueil ${ }^{24}$.

Quels charmes déploie ici cette évocation domestique ? C'est tout d'abord le caractère sériel qui donne du sens à l'image : la métaphore du ménage permet de décliner les différents vices qui menacent «l'habittation » de l'âme. Outre le plaisir de la cohérence qu'elle permet, elle sollicite la raison de l'auditeur pour la déchiffrer ${ }^{25}$. Enfin, la mise en œuvre du comparant invite le destinataire à évaluer la pertinence du

23 «Cet homme [...] est rendu égal aux bêtes de somme dépourvues d'intelligence et il devient semblable à elles. À sa suite, nous nous animalisons et, menant une vie de bétail, notre âme fléchissante s'abandonne inexorablement aux choses de la terre, nous dont la face, penchée à la manière des porcs, s'attache aux choses viles »: O. O., $n^{\circ} 208$, p. 15.

${ }^{24}$ Voir O. F., n ${ }^{\mathrm{O}} 340$, p. 433.

25 Voir Armand Strubel, Allégorie et littérature au Moyen Âge, op. cit. et Virginie Minet-Mahy, Esthétique et pouvoir de l'œuvre allégorique..., op. cit. 
référent et à apprécier, relativement, la maîtrise rhétorique du prédicateur. Peu importe que l'image soit raffinée : elle mobilise toujours le plaisir de l'imaginaire, la saveur de la chose vue et la jouissance esthétique face au remploi des topoi spirituels par le prédicateur.

La séduction des images, on l'a vu, ne vise pas seulement le plaisir esthétique : elle est aussi un outil didactique pour le prédicateur. Toutefois, Gerson ne s'interdit en rien de recourir à des procédés plus vigoureux pour jouer de la peur et donner du poids à la doctrine. C'est l'hypotypose qui permet dans cet exemple de conjuguer finalité pastorale (inspirer la peur du châtiment, et donc le repentir) et rhétorique spectaculaire en faisant surgir l'enfer sur la scène littéraire, et surtout mentale :

[...] Quidam impinguatus, incrassatus, dilatatus bene curata cute, unus Epicuri de grege porcus, viso altero poenitentia macilento et jejuno, he, miser, inquit, quid agis et unde venis ? Apparet quod venias de inferno. Cui ille non minus mundialiter: et tu inquit ad infernum pergere videris ${ }^{26}$.

Plutôt que de faire la critique de désordres moraux, Gerson transporte son auditeur dans l'au-delà en deux lignes ciselées. Les nominatifs rimés dépeignent en quelques mots un libertin médiéval et les allitérations en $[\mathrm{k}]$, rapprochant «curata cute» de «Epicuri » et «porcus» achèvent de porter la condamnation. Toutefois, l'évocation resterait plate et n'atteindrait pas son but sans le dialogue et la pointe comique et amère. Le sermon se transforme ainsi en vision qui s'impose à l'auditeur pour achever de le convaincre de la vanité des plaisirs.

26 «Un homme engraissé, encrassé, replet, la peau bien entretenue, un de ces pourceaux du troupeau d'Épicure s'exclame à la vue d'un autre amaigri par la pénitence et le jeûne: "Hé malheureux, que fais-tu et d'où viens-tu ? On dirait que tu viens de l'enfer". Et l'autre de répondre tout aussi courtoisement: "et toi tu sembles être arrivé en enfer". » O. O., n² 207, p. 11. 
La séduction des images n'est néanmoins que l'une des nombreuses ressources rhétoriques dont peut user le prédicateur car il est tout aussi pertinent de convoquer la persuasion de la chose sentie. Pour mieux prêcher la faute, l'art homilétique s'efforce de toucher son destinataire: Gerson use donc abondamment des émotions, les siennes propres ou celles des autres. Face au déploiement du pathos rhétorique, l'auditeur, saisi par l'empathie, adhère en même temps au contenu moral, comme quand Gerson donne la parole aux âmes du purgatoire :

[...] escoutez leur humble requeste et leur plainte doloreuse pour les aydier, secourir et delivrer.[...] entendez que dit la mere a son enfant ou chascun mort a son heritier ou executeur $[\ldots]$ : Mon chier enfant, dit la mere qui est en doloreuse prison de purgatoire, en peine et en tourment, mon chier enfant, entens a moy, regarde moy, escoute moy ${ }^{27}$.

L'auditeur est ici hélé par sa mère, ou son légataire, qui le supplie de lui faire quitter ce lieu. Comment se dérober à la détresse mise en scène et étayée par un réseau dense d'anaphores ? Gerson exploite en effet la richesse lexicale du français («requeste», «plainte», « secourir », «aidier»), ainsi que sa richesse sonore ([r], [ã]), pour donner à cette demande l'ampleur d'une supplique.

\section{Les charmes du matériau littéraire : effets narratifs et dramatisation}

Toutefois, le miel des images et des émotions ne peut pas toujours occulter l'amertume de la morale. Reste alors à se faire conteur ou dramaturge, le fond théologique restant, lui, peu affecté. La prédication des ordres mendiants, ou même celle des cisterciens ${ }^{28}$, le fait couramment

\footnotetext{
${ }^{27}$ O. F., n ${ }^{\circ} 344$, p. 553.

${ }^{28}$ Voir Faire croire..., op. cit.
} 
avec l'utilisation d'exempla; Gerson en revanche ne goûte guère ces pratiques.

Refus, donc, des récits qui faisaient le succès de certaines prédications, mais utilisation des ressources de la narrativité tout de même : les scènes à caractère allégorique permettent ainsi à Gerson de conjuguer narration, bienséance homilétique et pertinence théologique.

Ecce enim, si beati Gregorii et non Damasceni sententiae credimus Lucifer fuit supremus angelorum, et hic tamen a perfectione sua decidit, primus morbo peccati vulneratus interiit. [...] Hoc ut vidit Michael, zelo Dei accensus nec ferre valens ut alius honorem usurparet, primo disputativum et quasi scholasticum sive gymnasticum praelium cum eodem aggreditur [...] Habuit igitur Michael orationem hujusmodi : "Attende tibi, o Lucifer, et quid agas vide ${ }^{29}$.

La frontière entre scénographie allégorique et récit tiré des traditions apocryphes est ici mince: Lucifer est en effet associé clairement à l'orgueil, sujet du sermon, et s'apparente à une abstraction. Plus largement, les figures n'ont guère de consistance : peu décrites, elles servent avant tout de support à ce qui prend la forme d'une dispute scolastique. En fait de récit, le sermon déploie d'abord une altercation dialoguée entre deux entités éminemment symboliques. Elles sont toutefois intégrées dans un cadre narratif minimal comprenant une situation initiale («Lucifer fuit supremus angelorum») et un élément perturbateur (« hic tamen a perfectione sua decidit, primus morbo peccati

\footnotetext{
${ }^{29}$ «En effet, si nous nous appuyons sur les propos de saint Grégoire et non de Damascène, Lucifer fut le premier des anges, et malgré cela, celui-ci déchut de sa perfection : étant le premier à être atteint de la maladie du péché, il succomba. Quand Michel vit cela, enflammé du zèle divin et ne pouvant supporter qu'un autre usurpe son rang, il entama tout d'abord avec ce dernier une dispute qu'on qualifiera de scolastique, ou de compétition. Michel tint donc un discours à peu près de cet ordre : "Arrête-toi Lucifer, et vois ce que tu comptes faire." »O. O, $n^{0} 228$, p. 293.
} 
vulneratus interiit») qui tendent vers une résolution. La narration travaille donc le dynamisme du dialogue en transposant la théologie sous les traits de l'action oratoire ou guerrière.

Néanmoins, d'autres sermons de Gerson incluent des narrations plus fournies en personnages comme c'est le cas du Ad Deum vadit français. La narration y entrelace des récits bibliques, centrés autour du Christ, et des récits du chancelier, centrés sur Marie, qui offre un contrepoint féminin à la figure du Christ. Ses actions permettent d'incarner la théologie du dogme marial et de fournir des modèles dévotionnels, applicables par les fidèles laïcs comme dans l'exemple suivant :

Et vous, mere tres angoisseuse, les oyez patiemment, comme je tiens, et honnestement et constamment, comme il afferoit a une dame de telle vertus et constance. Et m'est assez a croire que vous consoliez plus les autres, en especial Marie Magdeleine, et les disciples, qu'on ne vous confortoit. Vous des lors commenciez estre conforteresse des desconfortes. Non pourquant sentiez vous par dedans vous griesves pointures de douleurs qui travailloient votre cœur maternel et tres beni ${ }^{30}$.

Les sermons de Gerson ne se contentent pas de mettre en récit le dogme : à la manière d'une méditation, ils proposent aussi à chacun de s'identifier dans des scénographies spirituelles. Histoire de l'âme pécheresse, histoire de conversion : autant de récits dont l'auditeur peut devenir le héros. Plus que la narration, c'est le recours à des dispositifs dramatiques, notamment le jeu des pronoms, qui inclut le destinataire dans le discours :

Cur ignava languescis, o anima christiana ? Quo pacto inerti segnitie languida deprimeris ? [...] Audis quod ad Deum vadit Jesus Christus et hic

${ }^{30}$ O. F., n 341 p. 478. 
crucifixus: eia ergo rumpe moras omnes, o anima $[. .$.

Sed Dices: «Debilitata sum, damnata sum, illaqueata sum, inviscata sum, incurvata sum, infixa sum in limo profundi $[\ldots]^{31} \gg$

Sous la rhétorique, le discours n'est rien d'autre qu'un acte de contrition. Mais l'échange dialogué convoque le fidèle en une scène intérieure. Le sermon se fait mise en mots d'un for intime chaotique, rehaussé d'une discrète mélancolie par les assonances et la prédication devient une véritable catharsis, ou conversion, guidée par le pas à pas du discours.

Le discours moral se prête à bien des enrichissements qui lui confèrent sa dimension esthétique, qu'il soit agrémenté de couleurs de rhétorique, ou mis en forme avec une scénographie narrative. Mais la prédication est également un discours oral ${ }^{32 .}$ C'est donc aussi en sa qualité de discours en chaire qu'il revêt un caractère artistique et qu'il participe de la culture intellectuelle de la fin du Moyen Âge : celle-ci attache en effet une importance marquée aux manifestations oratoires ${ }^{33}$.

L'utilisation de procédés polyphoniques par le sermon ${ }^{34}$ relève donc d'abord de cet arrière-plan intellectuel : la construction d'une scène oratoire est pleinement esthétique. Mais elle accroît aussi la force

31 «Ô âme chrétienne, pourquoi deviens-tu faible et sans énergie? Pourquoi te recroquevilles-tu mollement avec une morne apathie ? Entends : Jésus s'en est allé auprès de Dieu, là, il est crucifié. Allons donc, ne tarde plus, ô mon âme [...]. Mais tu réponds : "je suis faible, je suis condamnée, je suis ligotée, je suis engluée, je suis opprimée, je suis enlisée dans une boue profonde" [...]. » O. O., n ${ }^{0}$ 207, p. 9.

${ }^{32}$ La terminologie en matière d'étude des sermons pose quelques problèmes : en français le terme de « discours » ne renvoie qu'imparfaitement à un événement oral impliquant un texte. La terminologie anglaise utilisée notamment dans le cadre des sermon studies use, quant à elle, couramment du terme de performance qui allie l'idée d'événement daté et localisé à l'oralité.

33 Voir le traité contre Jean de Monzon: Gilbert Ouy, «La plus ancienne auvre retrouvée de Jean Gerson... », art. cit.

34 On parlera ici de procédés «polyphoniques » pour une pluralité de voix et non «dialogiques » dans une théorisation bakhtinienne. Le sermon, en effet, ne joue pas d'effets de polysémie ni d'ambiguïté du signe. 
rhétorique, la défense d'une position marquant plus que son affirmation. Les sermons gersoniens accueillent ainsi d'innombrables voix : celles des pécheurs, des âmes repentantes ou encore des vertus. Loin d'être un discours monologique, le sermon devient ainsi tantôt une quaestio, tantôt une plaidoirie qui montre le chancelier affrontant le Péché ou l'Erreur, avec courage et brio rhétorique :

Mais tantost par aventure on me dira le proverbe commun : Bien aise plaidoyer qui parle sans partie; car il semble nulz ne parle ou doye parler contre moy. [...] Mais helas autrement va. Je regarde que mile et mile advocaz, par lesquels j'entens les vices et pechiez font partie ont fait des pieça les gens de croire ceste verite $[\ldots]^{35}$.

Ces dispositifs permettent de ménager le suspense, le prédicateur pouvant toujours faire surgir un nouveau contradicteur. Notons toutefois que la polyphonie ne remet jamais en question la dimension univoque du dogme, l'orthodoxie étant toujours réaffirmée avec soin.

\section{L'éloquence morale : grand spectacle rhétorique et énonciation en sourdine}

L'animation du discours par des voix autres peut déboucher sur des pratiques reléguant le prédicateur à l'arrière-plan pour mettre en avant un énonciateur plus prestigieux. Il peut être nécessaire en effet, afin de rendre le discours moral audible, de le déléguer à d'autres instances investies d'une plus grande autorité spirituelle. Les allégories sont à cet égard des serviteurs fréquents pour Gerson; ces voix à la dimension universelle peuvent dire ce qu'il ne s'autorise pas à dire en son nom propre :

${ }^{35}$ O. F., $\mathrm{n}^{\mathrm{0}} 344$, p. 550. 
Properabat rursus quaerere studiositas cur non modo sit baptismus ut olim in nomine Jesu [...] dum haec inter loquendum superriruit zelus fervidus, filius caritatis : abscede hinc, ait o studiositas speculatrix, non est tempus disputandi sed plorandi [...]? Ecce imminuti sumus ex ira Dei effusa super nos plus quam gentes quae nomen Domini non noverunt neque invocaverunt ? Et ut alia praeteream, ecce quam exsecrando schismate Ecclesiam sponsam concuti sinit, dejici, porteri, rumpi, lacerari ${ }^{36}$.

Pour aborder la question du schisme papal, Gerson préfère laisser la parole à Curiosité Spéculative et à Zèle Fervent. Son discours est alors plus affectif, légitimé en cela par l'épithète «Fervent» qui caractérise l'allégorie, mais aussi plus engagé par référence au zèle qui donne son nom au personnage. Sans doute y a-t-il là une stratégie de dissimulation de la source de l'énonciation; mais l'entrelacement des énonciateurs s'inscrit également dans une stratégie de modulation du discours. Faire parler un autre, c'est aussi s'autoriser des tonalités autres, plus pathétiques ou plus véhémentes. Les allégories permettent de jongler avec les styles et les couleurs rhétoriques et participent du brio oratoire du sermon.

La même latitude dans les emplois est perceptible dans des procédés rhétoriques plus simples. Du point de vue de la légitimation du discours il peut ainsi être utile de faire parler une autorité comme saint

36 «Derechef la curiosité s'empressait de demander pourquoi on ne pratiquait plus le baptême en invoquant le seul nom de Jésus etc. Et tandis qu'elle discutait de ces sujets Zèle Fervent, le fils de Charité, fit irruption : "Arrière, Curiosité Spéculative, le temps n'est plus aux disputes mais aux larmes ! [...] Voici que nous menace la colère de Dieu qui s'abat sur nous, bien plus encore que sur les peuples qui ne connaissent ni n'invoquent le nom de Dieu ? Et, je passe sur le reste, voici qu'il laisse l'Église, son épouse ébranlée par ce schisme exécrable, il la laisse se faire déchirer, disperser, mettre en morceaux et en lambeaux." »O. O., $n^{\circ} 212$, p. 83. 
Bernard : «Avertite igitur paulisper mentem a me et ipsum Bernardum loqui putate, non $m e .{ }^{37} »$

Le procédé là encore est communicationnel et esthétique car le texte de Gerson pour la fête de saint Bernard en 1402 est aussi un pastiche littéraire qui joue avec le genre de l'homélie monastique. Légitimation rhétorique donc, mais aussi brio oratoire que cette reprise du vocabulaire sensuel du sermon sur le Cantique des cantiques, le public pouvant identifier le style du maître cistercien dans un jeu intertextuel ${ }^{38}$.

Au terme de ce bref parcours, comment définir la prédication? Quelle dimension culturelle revêt à l'extrême fin du Moyen Âge la prédication morale? Nous l'avons vu : prêcher la faute, c'est articuler dans une forme oratoire scolastique exposition du dogme et rappel de la morale. La prédication exclut donc une autonomie de la communication esthétique : ses textes sont fortement finalisés. Ne relevant pas de la culture laïque, elle participe en revanche pleinement de l'autre champ intellectuel qu'est la culture cléricale. Cette littérature n'est pas sans fondement idéologique, de même que la littérature laïque influencée par les valeurs aristocratiques. C'est donc dans ce cadre culturel spécifique que prêcher la faute peut être un art. En effet le discours du prédicateur convoque à la fois les ressources rhétoriques, alors à la base de la communication, et la sensibilité esthétique, dont Jean de Montreuil est un témoin, dans sa réception. Dans ce cadre culturel, le «plaisir du texte » se goûte au diapason du message, harmonieusement exprimé.

\footnotetext{
37 «Détachez un peu vos pensées de ma personne et imaginez que c'est Bernard qui parle, et non moi. »O. O., n ${ }^{\mathrm{O}} 230$, p. 326.

38 Sur les sermons monastiques, on se reportera aux travaux de Jean Leclercq, L'Amour des lettres et le désir de Dieu, op. cit.
} 